\title{
Surveillance for stage I testicular cancer: Maximizing benefit and minimizing harm
}

\author{
Piotr Czaykowski, MD, MSc, FRCPC \\ CancerCare Manitoba \& University of Manitoba, Winnipeg, MB, Canada
}

Cite as: Czaykowski P. Surveillance for stage I testicular cancer: Maximizing benefit and minimizing harm. Can Urol Assoc J 2022;16(2):34. http://dx.doi.org/10.5489/cuaj.7754

See related article on page 26

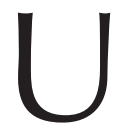
pdated Canadian consensus guidelines on the management of testicular germ cell cancer are forthcoming. Guideline authors agreed that surveillance is the preferred post-orchiectomy management option for all patients with stage I testicular cancer (seminoma or non-seminoma) assuming the patients' willingness and ability to adhere to a surveillance program; however, the guideline notes that optimal surveillance strategies have yet to be determined. ${ }^{1}$

The successful use of surveillance in early-stage testicular cancer is dependent on simultaneously maximizing the likelihood of identifying cancer recurrence as early as possible, while minimizing the risk of harm from the surveillance protocol. The harm most feared relates to second malignancies from ionizing radiation exposure during imaging investigations. Estimates of this risk vary widely in the literature, but it is not felt to be trivial, and is of greatest concern in those exposed at younger ages. ${ }^{2}$ Fortunately, advances in technology and technique have led to reduced radiation exposure per scan, and published experience, as well as a limited number of clinical trials, have gradually led to a reduction in recommended scan number and frequency without apparent compromise of patient outcomes. Additionally, exploration of imaging technology not associated with ionizing radiation (magnetic resonance imaging, ultrasound) has gained some momentum. As such, optimal surveillance imaging has proven to be a moving target.

In their article, Gyawali et al tackle an important question: in the real world, how closely do clinicians adhere to guideline recommendations for abdominal and chest imaging as part of the surveillance process for stage I testicular cancers. ${ }^{3}$ The data underpinning this analysis are from administrative datasets, and as such, represent an approximation of surveillance compliance. The study compares actual imaging usage to an Ontario benchmark (guidelines from the Princess Margaret Hospital $[\mathrm{PMH}])$, and notes both substantial underuse and overuse of imaging, defined as $>1$ scan under or over the recommended number in a given year of followup. Although it is difficult to discern consistent patterns emerging from the data, it is apparent that there is a frequent lack of concordance with the $\mathrm{PMH}$ guidelines. Given that the study is retrospective and relies on administrative data, it is not possible to ascertain the underlying reasons for under- and overuse of imaging relative to the $\mathrm{PMH}$ guidelines; it is also not possible to determine whether individual Ontario cancer centers based their surveillance protocol on the PMH guidelines, and thus were truly discordant.

Using this dataset, authors have previously shown that testicular cancer patients in Ontario have excellent outcomes, with no decrease in survival in more recent years, even as an initial surveillance approach for stage I disease has become more commonly used. ${ }^{4}$ The fact that frequent underuse of imaging relative to a benchmark doesn't appear to compromise survival is reassuring; however, that there seems to be considerable overuse is concerning, given this may represent a needless increased risk for patients.

Gywali et al's analysis suggests there is considerable variation in how surveillance for stage I testicular cancer is implemented and it points to an unmet need: evidence-informed, Canadian consensus guidelines providing a standardized approach to surveillance of stage I testicular cancer. This would allow more robust analysis of adherence to guidelines and would pave the way to exploring the root causes of significant deviation. Ultimately, this should lead to patient benefit, as we solve the simultaneous equations of maximizing benefit and minimizing harm.

Competing interests: The author does not report any competing personal or financial interests related to this work.

\section{References}

1. Hamilton RJ, Wood L, Canil C, et al. Canadian consensus guidelines for the management of testicular germ cell cancer. Can Urol Assoc J 2022. [Forthcoming]

2. Salminen $E$, Niniviita $H$, Jarvinen $H$, et al. Cancer death risk related to radiation exposure from computed tomography scanning among testicular cancer patients. Anticancer Res 2017;37:831-4. https://doi. org/10.21873/anticanres. 11385

3. Gyawali $B$, Griffiths R, Robinson AD, et al. Utilization of imaging for active surveillance in testicular cancer. Is real-world practice concordant with guidelines. Can Urol Assoc J 2022; 16:26-33. https://doi.org/10.5489/cuai.7246

4. Leveridge MJ, Siemens DR, Brennan K, et al. Temporal trends in management and outcomes of testicular cancer: A population-based study. Cancer 2018;124:2724-32. htrps://doi.org/10.1002/cncr.31390

Correspondence: Dr. Piotr Czaykowski, CancerCare Manitoba \& University of Manitoba, Winnipeg, MB, Canada; pczaykowski@cancercare.mb.ca 